\title{
Superheavy nuclei in self-consistent nuclear calculations
}

\author{
K. Rutz, ${ }^{1}$ M. Bender, ${ }^{1}$ T. Bürvenich, ${ }^{1}$ T. Schilling, ${ }^{1}$ P.-G. Reinhard, ${ }^{2,3}$ J. A. Maruhn, ${ }^{1,3}$ and W. Greiner ${ }^{1,3}$ \\ ${ }^{1}$ Institut für Theoretische Physik, Universität Frankfurt Robert-Mayer-Strasse 10, D-60325 Frankfurt, Germany \\ ${ }^{2}$ Institut für Theoretische Physik, Universität Erlangen Staudtstrasse 7, D-91058 Erlangen, Germany \\ ${ }^{3}$ Joint Institute for Heavy-Ion Research, Oak Ridge National Laboratory, P. O. Box 2008, Oak Ridge, Tennessee 37831 \\ (Received 22 October 1996)
}

\begin{abstract}
The shell structure of superheavy nuclei is investigated within various parametrizations of relativistic and nonrelativistic nuclear mean-field models. The heaviest known even-even nucleus ${ }_{156}^{264} \mathrm{Hs}_{108}$ is used as a benchmark to estimate the predictive value of the models. From that starting point, doubly magic spherical nuclei are searched in the region $Z=110-140$ and $N=134-298$. They are found at $(Z=114, N=184)$, $(Z=120, N=172)$, or at $(Z=126, N=184)$, depending on the parametrization. [S0556-2813(97)01305-8]

PACS number(s): 21.60.Jz, 21.30.Fe, 24.10.Jv, 27.90.+b
\end{abstract}

\section{INTRODUCTION}

The possible existence of islands of shell-stabilized superheavy nuclei has been an inspiring problem in heavy-ion physics for almost three decades [1]. Recent experiments at GSI $[2,3]$ and Dubna [4] brought innovations by producing isotopes at and in the vicinity of the deformed doubly magic nucleus ${ }_{162}^{270} \mathrm{Hs}_{108}$, as theoretically verified in macroscopicmicroscopic models $[5,6]$. The ultimate goal remains the spherical doubly magic superheavy nucleus ${ }_{184}^{298} 114$ which was predicted in the earliest macroscopic-microscopic investigations $[7,8]$ and confirmed in more recent models of this type $[5,6]$. The expectation that in the near future experimental progress will access this region is a strong motivation to investigate the shell structure of superheavy nuclei within the self-consistent nuclear mean-field models [9-11], especially since there were early indications [12] that proton and neutron shell closures strongly affect each other and that $Z=120$ may be a shell closure.

It is the aim of this contribution to scan a wide region of superheavy nuclei for the occurrence of spherical magic shells within the framework of the relativistic mean-field model (RMF) (for reviews see $[13,14]$ ) and within the nonrelativistic Skyrme-Hartree-Fock (SHF) approach (for a review see [15]).

The extrapolation towards superheavy nuclei challenges the predictive power of nuclear structure models. The macroscopic-microscopic method, although generally successful, requires preconceived knowledge about the expected densities and single-particle potentials, which fades away when stepping into new regions where stronger polarization effects and more complicated functional forms of the densities may occur. These effects are naturally incorporated within self-consistent nuclear models which nowadays manage to describe all known nuclei from ${ }^{16} \mathrm{O}$ on with satisfying quality by fixing a handful of model parameters [14,16,17]. There remain, however, several loosely fixed aspects in these parametrizations which amplify as uncertainties in extrapolations, e.g., to nuclei near the drip line [18] or to superheavy nuclei as discussed here.

\section{THE FRAMEWORK}

In view of the uncertainties, we consider a broad selection of parametrizations with about comparable quality concern- ing normal nuclear properties but differences in some detail. For the nonrelativistic SHF calculations we consider the parametrizations SkM* [19], SkI1 [17], SkP [20], SLy6 [16] which all employ the standard form but differ in bias. The force SkP uses effective mass $m^{*} / m=1$ and is designed to allow a self-consistent treatment of pairing. The other forces all have smaller effective masses around $m^{*} / m=0.7-0.8$. The force $\mathrm{SkM}^{*}$ was first to deliver acceptable incompressibility and fission properties and it is still a benchmark in this area. The force SLy6 stems from an attempt to cover properties of pure neutron matter together with normal nuclear ground-state properties; one can expect reliable extrapolations to neutron-rich nuclei from this force. The force SkI1 stems from a recent systematic fit (along the strategy of [21]) already embracing data from exotic nuclei; it is biased towards an optimal description of normal nuclei including surface properties.

The forces SkI3 and SkI4 are fitted exactly as SkI1 but using a variant of the Skyrme parametrization where the spin-orbit force is complemented by an explicit isovector degree-of-freedom [17]. They are designed to overcome the different isovector trends of spin-orbit coupling between conventional Skyrme forces and the RMF. SkI3 contains a fixed isovector part exactly analogous to the RMF, whereas SkI4 is adjusted allowing free variation of the isovector spinorbit force. Both forces contain a minimal relativistic correction within the SHF ansatz. The modified spin-orbit force has a strong effect on the spectral distribution in heavy nuclei and we expect visible consequences for the predictions of superheavy nuclei.

For the RMF we consider the parametrizations NL-Z [22], PL-40 [23], NL-SH [24], and TM1 [25]. The force NL-Z aims at a best fit to nuclear ground-state properties for the standard nonlinear ansatz [14] with cubic and quartic selfcoupling of the scalar field. The force PL-40 is a similar fit, but with a stabilized form of the scalar nonlinear selfcoupling. It shares most properties with NL-Z, as the good reproduction of ground-state properties and similar nuclear matter properties with the low effective mass $m * / m=0.58$ which is typical for the RMF. But PL-40 is somewhat more appropriate in the regime of small densities at the outer nuclear surface and thus yields better fission barriers [26]. The force NL-SH also employs the standard ansatz, but was 
TABLE I. Compilation of nuclear matter properties for the parameter sets used in this study. $E / A$ and $\rho_{0}$ denote the equilibrium energy per nucleon and density, $K_{\infty}$ the compression modulus, $m^{*}$ the effective mass (caution: defined differently for relativistic and nonrelativistic models [34]) and $a_{\text {sym }}$ the asymmetry coefficient. $\Delta r_{\mathrm{c}}^{2}$ is the isotope shift on charge r.m.s. radii for ${ }^{214} \mathrm{~Pb}-{ }^{208} \mathrm{~Pb}, \epsilon_{l s}$ the spin-orbit splitting between the $1 p_{3 / 2}$ and $1 p_{1 / 2}$ level in ${ }^{16} \mathrm{O}$, see [17].

\begin{tabular}{|c|c|c|c|c|c|c|c|c|}
\hline Force & $E / A[\mathrm{MeV}]$ & $\rho_{0}\left[\mathrm{fm}^{-3}\right]$ & $K_{\infty}[\mathrm{MeV}]$ & $m * / m$ & $a_{\mathrm{sym}}$ & $\Delta r_{\mathrm{c}}^{2}\left[\mathrm{fm}^{2}\right]$ & $\epsilon_{l s, p}[\mathrm{MeV}]$ & $\boldsymbol{\epsilon}_{l s, n}[\mathrm{MeV}]$ \\
\hline $\mathrm{SkM}^{*}$ & -16.01 & 0.160 & 217 & 0.789 & 30.0 & 0.359 & 6.2 & 6.3 \\
\hline SkP & -16.04 & 0.163 & 202 & 1.000 & 30.0 & 0.371 & 4.5 & 4.6 \\
\hline SLy6 & -15.92 & 0.159 & 230 & 0.690 & 32.0 & 0.428 & 5.7 & 5.8 \\
\hline SkI1 & -15.93 & 0.160 & 243 & 0.693 & 37.5 & 0.380 & 6.1 & 6.2 \\
\hline SkI3 & -15.96 & 0.158 & 258 & 0.577 & 34.8 & 0.567 & 6.3 & 6.3 \\
\hline SkI4 & -15.92 & 0.160 & 248 & 0.650 & 29.5 & 0.600 & 6.3 & 6.2 \\
\hline NL-Z & -16.19 & 0.151 & 174 & 0.58 & 41.8 & 0.650 & 5.8 & 5.8 \\
\hline PL-40 & -16.17 & 0.153 & 166 & 0.58 & 41.7 & 0.698 & 5.8 & 5.9 \\
\hline NL-SH & -16.33 & 0.146 & 355 & 0.66 & 36.1 & 0.587 & 6.8 & 6.9 \\
\hline TM1 & -16.3 & 0.145 & 281 & 0.634 & 36.9 & 0.646 & 5.6 & 5.7 \\
\hline Expt. & & & & & & 0.613 & 6.3 & 6.1 \\
\hline
\end{tabular}

adjusted with a bias to exotic nuclei, fitting neutron radii instead of surface thicknesses. Finally, the force TM1 includes a nonlinear self-coupling of the vector field as well, and is fitted in the same way as NL-SH.

In both, SHF and RMF, the pairing correlations are treated in the BCS scheme using a delta pairing force [16] $V_{\text {pair }}=V_{\mathrm{p} / \mathrm{n}} \delta\left(r_{1}-r_{2}\right)$. The strengths $V_{\mathrm{p}}$ for protons and $V_{\mathrm{n}}$ for neutrons depend on the actual mean-field parametrization. They are optimized by fitting (for each parametrization separately) the pairing gaps in Sn isotopes and the isotones with $N=82$. The pairing space was chosen twice as large as the given particle number with a smooth Fermi cutoff weight, for details see [27]. Furthermore, a center-of-mass correction is employed, for the SkI $x$, SLy6, NL-Z, and PL-40 forces by subtracting a posteriori $E_{\text {c.m. }}=\left\langle\hat{P}_{\text {c.m. }}^{2}\right\rangle / 2 m A$, for NL-SH and TM1 by subtracting the harmonic oscillator estimate $E_{\text {c.m. }}=\frac{3}{4} 41 A^{-1 / 3} \mathrm{MeV}$, while for SkM* and SkP only a diagonal correction is performed [21], as used in the original adjustment of these parameter sets.

The numerical procedure solves the coupled SHF and RMF equations on a grid in coordinate space with the damped gradient iteration method [28]. A spherical representation is employed in most of the calculations. An axially symmetric deformed representation has been used occasionally for counterchecks and particularly for the deformed system ${ }^{264} \mathrm{Hs}_{108}$.

To summarize the features of the forces subject to our investigation: all provide about the same good quality concerning the nuclear bulk properties, energies, and radii, in known stable nuclei. There are differences in surface properties: most forces perform very well in that respect, but the forces NL-SH and TM1 produce a too small surface thickness and correspondingly do not work so well in fission calculations; this holds, although less dramatically, for the force SLy6. There are differences in the effective mass: the modern fits SkI $x$, SLy6, NL-Z, and PL-40 all have low effective masses (below 0.7 for SHF and below 0.65 for the RMF models) whereas SkP even comes up to $m * / m=1$; this has consequences on the level density and thus on the shell structure in large systems. There are differences in the description of neutron-rich nuclei: the forces NL-SH and SLy6 are especially designed for this aspect, the forces SkIx include some information from the neutron-rich area in their fit, and the performance of all the other forces in that respect is yet untested. There are differences in isotopic trends of radii: all genuine SHF forces fail in that respect whereas RMF models do very well; the forces SkI3 and SkI4 use an extended Skyrme ansatz which manages to provide a good reproduction of the isotopic trends in charge radii of $\mathrm{Pb}$ isotopes. In that respect SkI4 is superior. Table I summarizes the nuclear matter properties of the forces discussed and gives an overview of the reproduction of the isotope shifts on charge radii in lead and the spin-orbit splitting in ${ }^{16} \mathrm{O}$.

\section{COMPARISON FOR AN EXISTING SUPERHEAVY NUCLEUS}

The question is now how all these parametrizations, which provide nearly comparable quality in the regime of known stable nuclei but differ in some details perform when extrapolating to the new area of superheavy nuclei. Before going into the regime of the yet unknown, we take the presently heaviest known nuclei as benchmarks. To that end we have calculated the ground states of the heaviest even-even nucleus for which the mass is known, i.e., ${ }_{156}^{264} \mathrm{Hs}_{108}$ [29]. This nucleus is close to a region of enhanced stability in the vicinity of the doubly deformed magic nucleus ${ }_{162}^{270} \mathrm{Hs}_{108}[5,6]$.

Table II shows ground-state properties of ${ }^{264} \mathrm{Hs}_{108}$ obtained from deformed mean-field calculations for the variety of forces explained above. The experimental binding energy is also given for comparison. The dimensionless multipole deformations are defined as $\beta_{\ell}=4 \pi\left\langle r^{\ell} Y_{\ell 0}\right\rangle /\left(3 A r_{0}^{\ell}\right)$ with $r_{0}=1.2 \mathrm{fm} A^{1 / 3}$ and provide a more immediate geometrical understanding than the multipole moments as such [30]. We see from Table II that almost all models agree in the predicted deformations, which corroborates the experience that 
TABLE II. Binding energy (in units of $\mathrm{MeV}$ ), relative error on binding energy, quadrupole deformation $Q_{2}$ in units of $\mathrm{fm}^{2}$, and dimensionless quadrupole $\left(\beta_{2}\right)$ and hexadecapole $\left(\beta_{4}\right)$ deformations for ${ }^{264} \mathrm{Hs}_{108}$ computed for several mean-field parametrizations as indicated in the first column. YPE+WS is the result of a macroscopic-microscopic calculation [5]. The last line shows the experimental binding energy from [29].

\begin{tabular}{lccccc}
\hline \hline Force & $E[\mathrm{MeV}]$ & $\delta E / E[\%]$ & $Q_{2}\left[\mathrm{fm}^{2}\right]$ & $\beta_{2}$ & $\beta_{4}$ \\
\hline SkM* & -1907.18 & 1.01 & 1033 & 0.28 & -0.01 \\
SkP & -1914.81 & 0.61 & 1053 & 0.28 & -0.01 \\
SLy6 & -1915.89 & 0.56 & 1034 & 0.28 & -0.02 \\
SkI1 & -1915.24 & 0.59 & 1057 & 0.28 & -0.02 \\
SkI3 & -1920.02 & 0.34 & 1020 & 0.27 & -0.02 \\
SkI4 & -1923.51 & 0.17 & 1012 & 0.27 & -0.02 \\
& & & & & \\
NL-Z & -1931.32 & -0.24 & 1074 & 0.29 & +0.00 \\
PL-40 & -1931.34 & -0.24 & 1072 & 0.29 & +0.00 \\
NL-SH & -1939.14 & -0.64 & 904 & 0.24 & +0.00 \\
TM1 & -1938.66 & -0.62 & 945 & 0.25 & 0.02 \\
& & & & & \\
YPE+WS & -1925.89 & 0.04 & & 0.24 & -0.03 \\
& & & & & \\
Expt. & -1926.72 & & & & \\
\hline \hline
\end{tabular}

well-developed deformations are a general topological feature of nuclear shell structure [8,31]. There is, however, a noteworthy exception in that NL-SH and TM1 produce a somewhat smaller quadrupole moment. It seems that their smaller surface thickness and larger effective mass modifies the shell structure so much that deformation properties are shifted. This feature is also found in fission barriers [26] and a systematic variation of the effective mass in studies of deformation energy surfaces [32].

The most interesting observable for our purposes is the binding energy, because experimental information is available. For better comparison, in the third column we display the relative errors between calculation and experimental value. Although all forces in our selection show acceptable quality in that extrapolated result, there are clearly visible differences. The Skyrme forces with the old standard spinorbit coupling have about the same error of about 0.6 , with recent fits coming a bit closer than older forces. The isovector-extended spin-orbit coupling in SHF produces a big step forward in quality concerning this observable, which shows that there is some truth in the relativistic isovector mix of the spin-orbit coupling. This is corroborated by the equally good results of the RMF forces NL-Z and PL-40. There is, however, a different sign in the error which hints at an essential difference between SHF and RMF, yet to be understood. The "exotic" RMF forces NL-SH and TM1 again fall below the quality of the more standard parametrizations. The conclusion from Table II is that for the extrapolations to superheavy nuclei, the forces SkI3, SkI4, NL-Z, and PL-40 should be preferred.

\section{SPHERICAL MAGIC SHELLS IN LARGER SUPERHEAVY NUCLEI}

The most interesting feature for even larger systems is the possible occurrence of new spherical doubly magic nuclei.
There are different possibilities for identifing magic numbers. One often considers a gap in the single-particle spectra as a signal for a magic number, but this is not always sufficient. In macroscopic-microscopic models the shell correction provides a natural measure for magicity. The shell correction is related to the difference between the experimental values of the nuclear masses and the predictions of a liquiddrop model. A more direct measure of a shell closure is the observation of a sudden jump in the two-nucleon separation energies $S_{2 p}(N, Z)=B(N, Z)-B(N, Z-2)$ for the protons or $S_{2 n}(N, Z)=B(N, Z)-B(N-2, Z)$ for the neutrons. Therefore the two-nucleon gaps

$$
\begin{gathered}
\delta_{2 p}(N, Z)=2 B(N, Z)-B(N, Z-2)-B(N, Z+2) \\
\delta_{2 n}(N, Z)=2 B(N, Z)-B(N-2, Z)-B(N+2, Z)
\end{gathered}
$$

show a pronounced peak for magic numbers [33]. We will consider the two-nucleon gaps (1) as the observable with large positive values indicating a shell closure. The scale of this quantity is indicated by the gaps for the doubly magic ${ }^{208} \mathrm{~Pb}$ which are $\delta_{2 p}=8.5 \mathrm{MeV}$ and $\delta_{2 n}=7.8 \mathrm{MeV}$ for SkI1. It is to be noted that the amplitude of shell effects decreases with increasing system size, due to the increasing level density. This will make it more and more difficult to find pronounced gaps for much larger systems.

When looking at shell gaps from spherical calculations we have to keep in mind that a guarantee for spherical shape can only be given for doubly magic nuclei where protons as well as neutrons experience a spherical shell closure. Singly magic nuclei have a good chance to stay spherical, but can deform occasionally. Only a deformed calculation can definitively decide in such cases the appropriate ground-state shape. Nonetheless, the spherical scan delivers certainly a reliable first orientation in the landscape of superheavy nuclei.

Figure 1 shows the proton and neutron gaps from spherical mean-field calculations with the chosen forces for a large variety of $Z$ and $N$. The results from force NL-Z are so close to those of PL-40 that we have displayed only one case. As expected, the largest gaps are much smaller than in the lead region (by about a factor of 2). In the following discussion we will consider the black squares (standing for the largest gaps) as indicators of a shell closure. The left column of Fig. 1 shows the proton gaps $\delta_{2 p}$. The isotopes of $Z=120$ have the most pronounced proton gaps in all cases, except for SkI4 where $Z=114$ is the preferred case, respectively SkM* and $\mathrm{SkP}$, where $Z=126$ is favored.

The right column of Fig. 1 shows the neutron gaps $\delta_{2 n}$. One sees a clear general trend in that the nonrelativistic models prefer a magic shell $N=184$ whereas the relativistic models tend towards a magic $N=172$ having, however, less pronounced magicity. The relativistic forces PL-40 and NL-Z have hints to magicity in both shells, 172 as well as 184 . For $Z<110$ also NL-SH develops a shell closure at $N=184$ as shown in [11]. Generally, it is to be noted that those four forces which are preferred from comparison with ${ }^{264} \mathrm{Hs}_{108}$ produce the best developed shell closures for protons, whereas in all standard SHF models (SLy6, SkI1, $\mathrm{SkM}^{*}, \mathrm{SkP}$ ) and the relativistic NL-SH as well as TM1 the shell structure appears to be less pronounced. The more re- 


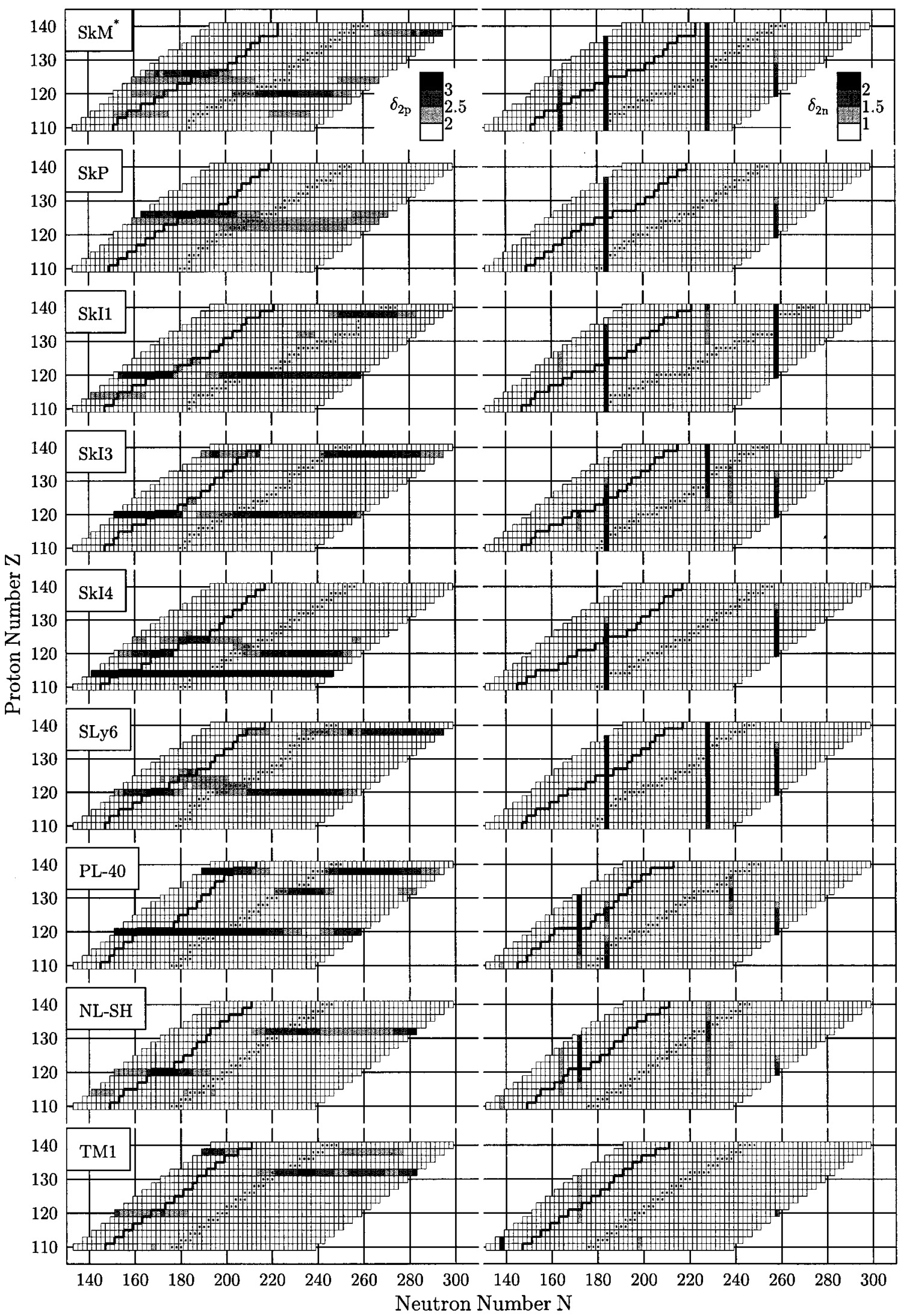

FIG. 1. Grey scale plots of proton gaps (left column) and neutron gaps (right column) in the $N-Z$ plane for spherical calculations with the forces as indicated. The assignment of scales differs for protons and neutrons, see the uppermost boxes where the scales are indicated in units of $\mathrm{MeV}$. Nuclei that are stable with respect to $\beta$ decay and the two-proton dripline are emphasized. 


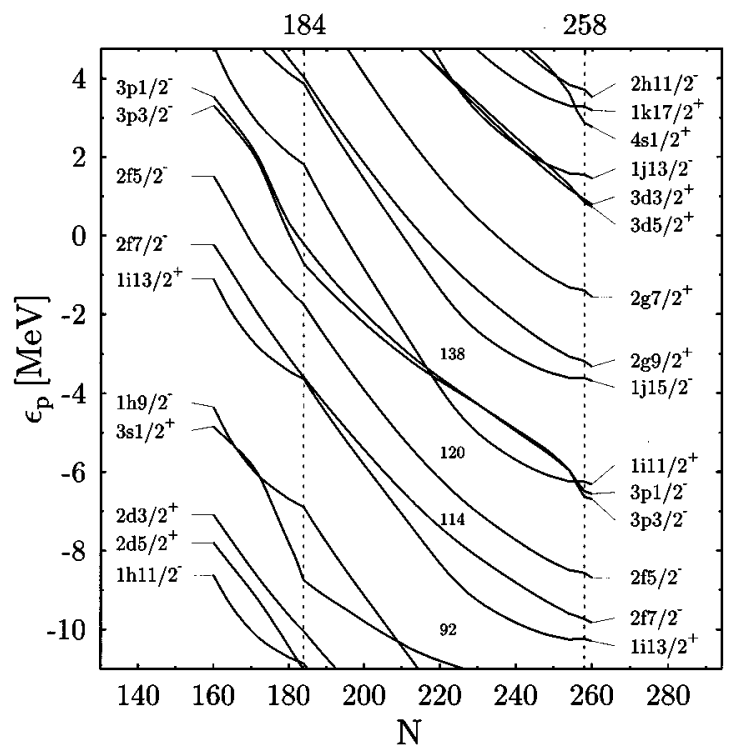

FIG. 2. The single proton levels near the Fermi energy for the isotopes of $Z=120$ versus the neutron number, computed with SkI1. Due to minimal relative changes of the single proton levels the proton gap at $Z=120$ vanishes in the vicinity of $N=184$, the neutron number where the proton shell gap $\delta_{2 p}$ is lowest, see Fig. 1 .

liable forces thus prefer shell closures and this hints that some magic system will be observed in that range of nuclei.

The most interesting species are, of course, the doubly magic systems. These require a simultaneous occurrence of a large shell gap (black squares) for the protons (left column) as well as for the neutrons (right column). It is interesting to note that such a coincidence is not trivial, as we see from the many cases where it cannot be found (SkI1, SkI3, SLy6, TM1). The remaining parametrizations do predict doubly magic nuclei, however, at different places. The forces SkP and $\mathrm{SkM}^{*}$ predict $Z=126, N=184$. The preselection with ${ }^{264} \mathrm{Hs}_{108}$ has picked the two forces SkI4 and PL-40 (=NL-Z) both of which show doubly magic nuclei. The relativistic PL-40 parametrization predicts $Z=120, N=172$, whereas the nonrelativistic SkI4 prefers $Z=114, N=184$. Thus even two optimized and preselected forces make conflicting predictions. It is to be noted that shell models usually predict the doubly magic $Z=114, N=184$ [5-8]. The more robust occurrence of the magic $N=184$ neutron shell and the more favorable charge asymmetry seem to indicate a preference for this configuration.

We prefer, however, to read the result the other way round. The study of superheavy nuclei has disclosed significant deviations amongst a set of otherwise comparable mean-field models. In particular, there is a systematic difference between the RMF and SHF models which has yet to be understood. New experimental information on superheavy nuclei will help to clarify these open theoretical questions.

One sees in Fig. 1 that the proton shell closures for a given $Z$ can change with varying neutron number, and similarly the neutron shell closures vary with changing proton numbers. A vivid example is the $Z=120$ shell computed with SkI1 which starts with closure, loses that property with increasing neutron number, and regains it later. The changes are related to a changing level density at the Fermi surface. As a demonstration, we show in Fig. 2 the single proton spectra for this case, i.e., $Z=120$ computed with SkI1. One has to watch the shell gap at $Z=120$. Minimal relative changes of the single proton levels indeed produce a regime of higher level densities around $N=184$, the neutron number where the proton shell gap is lowest, see Fig. 1. This example illustrates that shell closures in superheavy nuclei are an extremely sensitive property. It is no surprise that this question imposes severe constraints on models and forces.

\section{CONCLUSIONS}

We have investigated the description of superheavy nuclei in the framework of relativistic and nonrelativistic nuclear mean-field models. A representative selection of parametrizations is considered which provide all about the same good quality concerning nuclear bulk observables but differ with respect to surface tension, effective mass, and isovector features. We take advantage of the heaviest experimentally measured even-even nucleus and use its binding energy to check the predictive power of the preselected forces. This shows a clear preference for the standard relativistic forces (NL-Z, PL-40) and relativistically corrected Skyrme forces (SkI4, SkI3). Shell closures are quantified in terms of the shell gap, i.e., the second difference of binding energies. A systematic survey of shell gaps in the range of $110<Z<140$ and $134<N<298$ shows that the preferred forces also provide more pronounced shell closures. There remain, however, conflicting predictions for a doubly magic system: $Z=120, N=172$ for the relativistic forces PL-40, NL-Z, and NL-SH but $Z=114, N=184$ for the nonrelativsitic force SkI4 and $Z=126, N=184$ for the standard Skyrme forces SkM* and SkP. Additional criteria (general trends, shell model predictions, charge asymmetry) set a preference on the case $Z=114, N=184$. But the conclusion is rather that the study of superheavy systems remains a challenge for self-consistent nuclear mean-field models, which have to be developed to a new stage by much more rigorous testing of a wide variety of nuclear properties throughout the periodic table. In particular the results have revealed a systematic difference between the relativistic and the nonrelativistic models which deserves further close inspection.

\section{ACKNOWLEDGMENTS}

The authors would like to thank S. Hofmann, G. Münzenberg, and D. Habs for many valuable discussions. This work was supported by Bundesministerium für Bildung und Forschung (BMBF) Deutsche Forschungsgemeinschaft (DFG), Gesellschaft für Schwerionenforschung (GSI), and by Graduiertenkolleg Schwerionenphysik. The Joint Institute for Heavy Ion Research has as member institutions the University of Tennessee, Vanderbilt University, and the Oak Ridge National Laboratory; it is supported by the members and by the Department of Energy through Contract No. DE-FG0587ER40361 with the University of Tennessee. 
[1] K. Kumar, Superheavy Elements (Adam Hilger, Bristol, 1989).

[2] S. Hofmann, V. Ninov, F. P. Hessberger, P. Armbruster, H. Folger, G. Münzenberg, H. J. Schött, A. G. Popeko, A. V. Yeremin, A. N. Andreyev, S. Saro, R. Janik, and M. Leino, Z. Phys. A 350, 277 (1995); 350, 281 (1995).

[3] S. Hofmann, V. Ninov, F. P. Hessberger, P. Armbruster, H. Folger, G. Münzenberg, H. J. Schött, A. G. Popeko, A. V. Yeremin, S. Saro, R. Janik, and M. Leino, Z. Phys. A 354, 229 (1996).

[4] Yu. A. Lazarev, Yu. V. Lobanov, Yu. Ts. Oganessian, V. K. Utyonkov, F. Sh. Abdullin, A. N. Polyakov, J. Rigol, I. V. Shirokovsky, Yu. S. Tsyganov, S. Iliev, V. G. Subbotin, A. M. Sukhov, G. V. Buklanov, B. N. Gikal, V. B. Kutner, A. N. Mezentsev, K. Subotic, J. F. Wild, R. W. Lougheed, K. J. Moody, Phys. Rev. C 54, 620 (1996).

[5] Z. Patyk and A. Sobiczewski, Nucl. Phys. A533, 132 (1991).

[6] P. Möller and J. R. Nix, Nucl. Phys. A549, 84 (1992); J. Phys. G 20, 1681 (1994).

[7] U. Mosel and W. Greiner, Z. Phys. 222, 261 (1969).

[8] S. G. Nilsson, C. F. Tsang, A. Sobiczewski, Z. Szymanski, S. Wycech, C. Gustafson, I.-L. Lamm, P. Möller, and B. Nilsson, Nucl. Phys. A131, 1 (1969).

[9] S. Ćwiok, J. Dobaczewski, P.-H. Heenen, P. Magierski, and W. Nazarewicz, Nucl. Phys. A611, 211 (1996).

[10] J.-F. Berger, L. Bitaud, J. Decharge, M. Girod, and S. PeruDessenfants, Proceedings of the International Workshop XXXIV on Gross Properties of Nuclei and Nuclear Excitations, Hirschegg, Austria, January, 1996 (GSI, Darmstadt, 1996).

[11] G. A. Lalazissis, M. M. Sharma, P. Ring, and Y. K. Gambhir, Nucl. Phys. A608, 202 (1996).

[12] M. Beiner, H. Flocard, M. Vénéroni, and P. Quentin, Phys. Scr. 10A, 84 (1974).

[13] B. D. Serot and J. D. Walecka, Adv. Nucl. Phys. 16, 1 (1986).

[14] P.-G. Reinhard, Rep. Prog. Phys. 52, 439 (1989).
[15] P. Quentin and H. Flocard, Annu. Rev. Nucl. Part. Sci. 28, 523 (1978).

[16] E. Chabanat, P. Bonche, P. Haensel, J. Meyer, and R. Schaeffer (unpublished).

[17] P.-G. Reinhard and H. Flocard, Nucl. Phys. A584, 467 (1995).

[18] W. Nazarewicz, J. Dobaczewski, T. R. Werner, J. A. Maruhn, P.-G. Reinhard, K. Rutz, C. R. Chinn, A. S. Umar, and M. R. Strayer, Phys. Rev. C 53, 740 (1996).

[19] J. Bartel, P. Quentin, M. Brack, C. Guet, and H.-B. Håkansson, Nucl. Phys. A386, 79 (1982).

[20] J. Dobaczewski, H. Flocard, and J. Treiner, Nucl. Phys. A422, 103 (1984).

[21] J. Friedrich and P.-G. Reinhard, Phys. Rev. C 33, 335 (1986).

[22] M. Rufa, P.-G. Reinhard, J. A. Maruhn, W. Greiner, and M. R. Strayer, Phys. Rev. C 38, 390 (1989).

[23] P.-G. Reinhard, Z. Phys. A 329, 257 (1988).

[24] M. M. Sharma and P. Ring, Phys. Rev. C 45, 2514 (1992).

[25] Y. Sugahara and H. Toki, Nucl. Phys. A579, 557 (1994).

[26] K. Rutz, J. A. Maruhn, P.-G. Reinhard, and W. Greiner, Nucl. Phys. A590, 680 (1995).

[27] M. Bender, P.-G. Reinhard, K. Rutz, and J. A. Maruhn (unpublished).

[28] V. Blum, G. Lauritsch, J. A. Maruhn, and P.-G. Reinhard, J. Comput. Phys. 100, 364 (1992).

[29] G. Audi and A. H. Wapstra, Nucl. Phys. A595, 409 (1995).

[30] R. W. Hasse and W. D. Myers, Geometrical Relationships of Macroscopic Nuclear Physics (Springer, Berlin, 1988).

[31] M. Brack, J. Damgård, A. S. Jensen, H. C. Pauli, V. M. Strutinsky, and C. Y. Wong, Rev. Mod. Phys. 44, 320 (1972).

[32] V. Blum, J. A. Maruhn, P.-G. Reinhard, and W. Greiner, Phys. Lett. B 323, 262 (1994).

[33] W. Nazarewicz, J. Dobaczewski, and T. R. Werner, Phys. Scr. T56, 9 (1995).

[34] M. Jaminon and C. Mahaux, Phys. Rev. C 40, 354 (1989). 